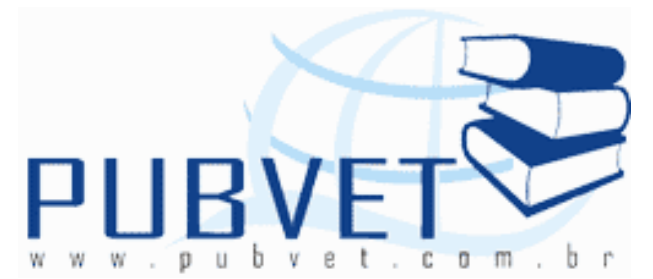

PUBVET, Publicações em Medicina Veterinária e Zootecnia.

\title{
Toxocaríase em crianças e adolescentes: uma revisão sistemática dos estudos epidemiológicos
}

Ingrid Ney Kramer de Mello ${ }^{1}$, Pricila A. Grasse Pietralonga ${ }^{2}$

${ }^{\mathbf{1}}$ Mestranda em Medicina Veterinária, Laboratório de Parasitologia, Departamento de Veterinária, Universidade Federal de Viçosa, Viçosa, MG.

${ }^{2}$ Mestranda em Medicina Veterinária, Laboratório de Doenças Bacterianas, Departamento de Veterinária, Universidade Federal de Viçosa, Viçosa, MG

\section{Resumo}

A presente revisão sistemática descreve a prevalência e os fatores de riscos associados à soroprevalência positiva na faixa etária de zero a 18 anos. A metodologia utilizada foi pesquisa em base de dados como Pubmed, Science direct e Scielo. Foram selecionados 91 artigos inicialmente, os quais 13 foram considerados relevantes para o presente trabalho. Em todos os trabalhos analisados, foi grande a variação da prevalência da doença. As condições socioeconômicas da família juntamente com ter contato com cães e gatos e areia foram os principais fatores de riscos para toxocaríase, e outras variáveis como onicofagia, geofagia, não lavar as mãos antes de comer também foram identificados como fatores de risco e alguns autores associaram sinais clínicos à soroprevalência positiva. Concluiu-se que são necessários mais estudos de base populacional representativos dessas faixas etárias e critérios uniformes para 
definir a prevalência e os fatores de risco, além da necessidade de medidas preventivas.

Palavras-chave: Toxocara canis, fatores de risco, crianças.

\title{
Toxocariasis in children and adolescents: a systematic review of epidemiological studies
}

\begin{abstract}
This systematic review describes the prevalence and risk factors associated with seroprevalence in age from zero to 18 years. The research methodology was used in the database as PubMed, Science Direct and Scielo. 91 articles were selected initially, which 13 were considered relevant to this work. In all studies reviewed, it was great variation in disease prevalence. The socioeconomic conditions of families with no contact with cats and dogs and sand were the main risk factors for toxocariasis, and other variables such as nail biting, geophagy, not washing hands before eating were also identified as risk factors and some authors associated seroprevalence of positive signs. It was concluded that further population based studies representative of these age groups and uniform criteria to define the prevalence and risk factors, besides the need for preventive measures.
\end{abstract}

Keywords: Toxocara canis, risk factors, children.

\section{INTRODUÇÃO}

A toxocaríase ocorre devido à infecção por larvas de Toxocara canis e Toxocara cati, nematódeos pertencentes à família Ascaridae. As formas adultas desses parasitas residem no trato gastrintestinal de seus hospedeiros definitivos, cães e gatos, respectivamente (REY, 2008). A infecção nos cães é observada com maior freqüência em cadelas prenhes e lactantes, assim como, em filhotes onde a contaminação ocorre por via transplacentária e 
MELLO, I.N.K. e PIETRALONGA, P.A.G. Toxocaríase em crianças e adolescentes: uma revisão sistemática dos estudos epidemiológicos. PUBVET, Londrina, V. 5, N. 31, Ed. 178, Art. 1203, 2011.

transmamária, sendo que as maiores prevalências de carga parasitária são observadas em filhotes na idade de três a seis meses (DAMIAN et al. 2007).

Nesses animais, as fêmeas adultas no intestino delgado são capazes de produzir até 200.000 ovos diariamente, que são eliminados juntamente com as fezes dos animais, tornando-se infectantes em duas a cinco semanas, sob condições de temperatura e umidade adequadas (SCHANTZ, 1989).

Uma vez presentes no solo, os ovos larvados podem ser ingeridos acidentalmente pelo ser humano. Ao serem ingeridos, vão para o intestino delgado e migram através dos órgãos somáticos, preferencialmente o fígado e os olhos, levando a duas diferentes formas clássicas: Larva migrans visceral (VLM) e a larva migrans ocular (OLM). A forma VLM, podem ocorrer distúrbios respiratórios como tosse persistente e asma (ALDERETE et al. 2003; TONELLI, 2005), efusão pleural (ASHWATH et al. 2004), e dermatopatias como prurigo, urticária, eczema (GAVIGNET, et al. 2008) e abcessos piogênicos (RAYES et al. 2001). Há ainda relatos de alterações neurológicas, incluindo convulsões (MOREIRA-SILVA et al. 2004), Meningoencefalite (VIDAL et al. 2003) e epilepsia (BÄCHLI et al. 2004). A forma OLM leva a quadro de uveíte e lesões ópticas (PAWLOWSKI, 2001; AZAR et al. 2004).

A toxocaríase é uma zoonose de distribuição mundial e considerada a helmintíase mais prevalente em países industrializados, entretanto, é pouco reconhecida como problema de saúde pública (MAGNAVAL et al. 2001; ALTCHEH et al. 2003).

Os aspectos clínicos da síndrome no homem são variáveis e dependentes de fatores como resposta imune do hospedeiro, padrão de migração, dose infectante e distribuição das larvas nos diferentes tecidos (SCHANTZ, 1989). Na grande maioria dos casos comporta-se como uma doença benigna de curso limitado, embora existam casos graves e fatais (CHIEFFI et al. 1990).

Segundo Rey (2008), a convivência com os animais hospedeiros não é essencial para contrair a doença. Entretanto, estudos recentes têm demonstrado ovos embrionados de $T$. canis no pêlo de cães (WOLF; WRIGHT, 
2004; KEEGAN; HOLLAND, 2010). Desta forma, o contato direto com cães pode ser considerado uma forma de transmissão. Outra modalidade de transmissão ao homem, porém mais rara, é a ingestão de carne crua ou mal cozida de hospedeiros paratênicos do helminto como coelhos, suíno, ovino e frango (STÜRCHLER et al. 1990; SALEM; SCHANTZ, 1992; FAN et al. 2004; MORIMATSU et al. 2006).

O diagnóstico da infecção humana por $T$. canis é feito por métodos indiretos, com detecção de níveis elevados de anticorpo IgG anti-Toxocara canis no sangue ou fluídos biológicos dos pacientes. Estes anticorpos podem ser detectados por diversas técnicas; dentre as mais utilizadas está o método ELISA. Atualmente, no teste ELISA tem sido utilizado antígeno ES da larva adsorvido do soro com antígeno de Ascaris sum, que mostra sensibilidade de $78 \%$ e especificidade de $92 \%$, sendo mais indicado do que o teste que utiliza o antígeno total da larva, pois pode haver reação cruzada com leishmaniose, esquistossomose, doença de Chagas, malária, ascaridíase, dentre outras patologias tropicais (CAMPOS JUNIOR et al. 2003; DAMIAN et al. 2007).

As crianças compõem o principal grupo de risco de infecção, devido aos hábitos geofágicos, onicofágicos e de brincar em contato com o solo (THOMASAMBROISE, 2000; GAWOR et al. 2008). A presente revisão sistemática tem como objetivo identificar a prevalência e os fatores de risco para toxocaríase em crianças e adolescentes em diferentes regiões do mundo.

\section{MATERIAIS E MÉTODOS}

\section{1- Estratégias de busca}

As bases de dados utilizadas na etapa de busca foram PubMed (<http://www.ncbi.nlm.nih.gov/pubmed $>$ ), Science direct (<http:/www. sciencedirect.com $>$ ) e SciELO (<http://www.scielo.org/php/index.php $>$ ). A pesquisa foi limitada a artigos publicados anteriormente a setembro de 2010 
(quando a pesquisa foi iniciada). As palavras chaves utilizadas na busca foram: "Toxocara", "crianças", "prevalência" e "fatores de risco". Os descritos em inglês foram "Toxocara", "children", "prevalence" and "risk factors". Todos os títulos e resumos (quando disponíveis) de cada pesquisa foram examinados e, em seguida os artigos pertinentes foram obtidos para análise. As referencias bibliográficas dos artigos foram examinadas para possíveis referencias adicionais. Não houve restrições quanto ao local do estudo ou idioma de publicação.

A etapa de busca selecionou inicialmente 91 artigos. Os critérios de inclusão foram: (1) população com idade de zero a 18 anos, amostra representativa da população escolar e hospitalar; (2) ser um estudo com delineamento epidemiológico que demonstrasse os fatores de risco e prevalência para a toxocaríase em crianças e adolescentes. Baseados nesses critérios foram selecionados 13 artigos. Os trabalhos foram obtidos através do portal de periódicos da CAPES.

\section{RESULTADOS}

\section{1- Prevalências de toxocaríase em crianças}

No Brasil foram selecionados seis estudos, todos utilizaram delineamento transversal (três base escolar e três base hospitalar) com exceção de apenas um que utilizou delineamento de coorte após estudo transversal. De todos os demais países obtiveram-se sete estudos, destes, seis eram de delineamento transversal, (três base escolar, três base Hospitalar) e um estudo casocontrole realizado nos Estados Unidos. $\mathrm{Na}$ tabela 1 , estão listadas as prevalências de toxocaríase por estudo selecionado. No Brasil, as prevalências variaram entre 8,7 a 38,8\% (ALDERETE et al. 2003; CAMPOS JúNIOR et al. 2003; COELHO et al. 2004; TEIXEIRA et al. 2006; PALUDO et al. 2007; CORREA; BISMARCK, 2010), com ampla diversidade de regiões e faixa etária 
MELLO, I.N.K. e PIETRALONGA, P.A.G. Toxocaríase em crianças e adolescentes: uma revisão sistemática dos estudos epidemiológicos. PUBVET, Londrina, V. 5, N. 31, Ed. 178, Art. 1203, 2011.

entre 0 a 18 anos. Nos demais países as prevalências variaram entre 25 a $67 \%$ (SADJJADI et al. 2000; BABOOLAL; RAWLINS, 2002 ; LOPEZ et al. 2007; ROLDÁN et al. 2008; SVIBEN et al. 2009; SHARIF et al. 2010).

Tabela 1. Prevalência de toxocaríase em vários países de 2002 a 2010.

\begin{tabular}{|c|c|c|c|c|}
\hline Países & $\begin{array}{c}\mathrm{n}^{\circ} \\
\text { crianças }\end{array}$ & Delineamento & Idade & Prevalência \\
\hline \multicolumn{5}{|l|}{ Brasil } \\
\hline Alderete et al. (2003) & 494 & $\begin{array}{c}\text { Transversal base } \\
\text { escolar }\end{array}$ & 7 a 16 anos & $38,8 \%$ \\
\hline $\begin{array}{l}\text { Campos Júnior et al. } \\
\text { (2003) }\end{array}$ & 602 & $\begin{array}{c}\text { Transversal base } \\
\text { hospitalar e } \\
\text { ambulatorial }\end{array}$ & 1 a 12 anos & $\begin{array}{c}\text { Grupo A } \\
(21,8 \%) ; \\
\text { Grupo B } \\
(9 \%)\end{array}$ \\
\hline Coelho et al. (2004) & 180 & $\begin{array}{c}\text { Transversal base } \\
\text { escolar }\end{array}$ & 3 a 7 anos & $38,3 \%$ \\
\hline Teixeira et al. (2006) & 242 & $\begin{array}{c}\text { Transversal base } \\
\text { hospitalar }\end{array}$ & $1-15$ anos & $8,7 \%$ \\
\hline Paludo et al. (2007) & 450 & $\begin{array}{c}\text { Transversal base } \\
\text { hospitalar }\end{array}$ & $\begin{array}{c}\text { Sete meses a } \\
12 \text { anos }\end{array}$ & $28,8 \%$ \\
\hline $\begin{array}{c}\text { Correa e Bismarck } \\
(2010) \\
\text { Argentina }\end{array}$ & 100 & $\begin{array}{l}\text { Transversal base } \\
\text { escolar e coorte }\end{array}$ & 6 a 14 anos & $28 \%$ \\
\hline Lopez et al. (2007) & 182 & $\begin{array}{c}\text { Transversal base } \\
\text { hospitalar }\end{array}$ & $\begin{array}{l}\text { Zero a } 16 \\
\text { anos }\end{array}$ & $67 \%$ \\
\hline $\begin{array}{c}\text { Irã } \\
\text { Sadjjadi et al. (2000) }\end{array}$ & 519 & $\begin{array}{c}\text { Transversal base } \\
\text { hospitalar }\end{array}$ & 6-13 anos & $25,6 \%$ \\
\hline Sharif et al. (2010) & 1210 & $\begin{array}{c}\text { Transversal base } \\
\text { escolar }\end{array}$ & 7 a 14 anos & $25 \%$ \\
\hline $\begin{array}{c}\text { Trinidade } \\
\text { Baboolal e Rawlins } \\
\text { (2002) } \\
\text { Peru }\end{array}$ & 1009 & $\begin{array}{c}\text { Transversal base } \\
\text { escolar }\end{array}$ & 5 a 12 anos & $62,3 \%$ \\
\hline Roldán et al. (2008) & 646 & $\begin{array}{l}\text { Transversal base } \\
\text { escolar e coorte }\end{array}$ & 5 a 12 anos & $31,1 \%$ \\
\hline $\begin{array}{c}\text { Croácia } \\
\text { Sviben et al. (2009) }\end{array}$ & 142 & $\begin{array}{c}\text { Transversal base } \\
\text { hospitalar }\end{array}$ & 3 a 18 anos & $31 \%$ \\
\hline
\end{tabular}


Os estudos de Baboolal e Rawlins (2002) e Sharif et al. (2010), revelaram associação significativa entre soroprevalência positiva e o sexo masculino. Ambos autores justificam esta exposição ao comportamento social observado geralmente em meninos nesta faixa etária como brincar em áreas não pavimentadas, praças e campos de areia. Nos demais estudos não houve relação significativa entre os sexos (SADJJADI et al. 2000; ALDERETE et al. 2003; TEIXEIRA et al. 2006; PALUDO et al. 2007; LOPEZ et al. 2007; SVIBEN et al. 2009). Não existindo, portanto uma consistência entre os estudos quanto à diferença de prevalência entre os sexos.

Paludo et al. (2007) mostraram em seu trabalho que o aumento da prevalência esta associado a idade (sete meses a cinco anos). Contudo os demais autores relataram não haver associação positiva entre soroprevalência e a idade.

\section{2- Fatores de risco para a toxocaríase}

Os fatores de risco identificados pelos autores foram contato com areia, a posse de cães ou gatos, quintal com terra e jardim, nível socioeconômico, onicofagia, geófagia, local de residência e não lavar as mãos antes de comer (tabela 2).

Na literatura, a condição sócio econômica das famílias foi freqüentemente identificada como fator de risco para a soroprevalência positiva para Toxocara sp., embora nem sempre isso tenha sido demonstrado. Alderete et al. (2003) e Paludo et al. (2007), observaram que renda familiar menor a cinco salários mínimos foi considerado como perfil de risco determinantes para a aquisição da infecção. LOPEZ et al.(2007), na Argentina das 182 crianças estudadas, 122 foram positivas (67\%), 28,8\% não tinham água potável em casa, 58,8\% não tinham rede de esgoto, e $86,7 \%$ viviam em ruas não pavimentadas. Nesse sentido Baboolal e Rawlins (2002) e Coelho et al. (2004), também encontraram 
MELLO, I.N.K. e PIETRALONGA, P.A.G. Toxocaríase em crianças e adolescentes: uma revisão sistemática dos estudos epidemiológicos. PUBVET, Londrina, V. 5, N. 31, Ed. 178, Art. 1203, 2011.

associação entre sorologia positiva e ausência de água encanada no domicílio e ruas não pavimentadas, respectivamente.

Em inquérito realizado em Brasília, por Campos Júnior et al. (2003), houve influencia dos fatores socioeconômicos sobre a freqüência de anticorpos nas crianças, com diferença significativa na população de bairros pobres e atendidas pelo sistema único de saúde $(21,8 \%)$ em relação `aquela que residia em bairros mais ricos da cidade e providas de planos de saúde particular (3\%). Segundo os autores, as crianças que viviam na periferia eram mais expostas à infecção por morarem em locais desprovidos de infra-estrutura sanitária, em casa de construção precária, sem condições adequadas de higiene, e por terem contato com grande quantidade de cães. Todavia, os autores não avaliaram os fatores de risco.

Sadjjadi et al. (2000) demonstrou em seu trabalho que crianças com pais analfabetos apresentaram maior soroprevalência em relação a crianças com pais alfabetizado e ainda que população da área urbana revelou maior prevalência em relação a áreas rurais. Resultado diferentemente relatado por Baboolal e Rawlins (2002). Entretanto, Sharif et al. (2010) relataram não haver associação entre soropositividade e classe social da família.

O contato com cães ou gatos e foram considerados fatores de risco para soroprevalência positiva (TEIXEIRA et al. 2006; PALUDO et al. 2007; SHARIF et al. 2010). Em Análise de regressão logística de 155 pares caso-controle foi demonstrado elevado riscos relativos $(R R=5,22)$ para ter tido uma ninhada de cachorros em casa (MARMOR et al. 1987) e além disso Baboolal e Rawlins (2002) encontraram associação positiva em ter cães em casa com sorologia positiva para Toxocara sp. Todavia Sadjjadi et al. (2000) e Roldán et al.(2009) não encontraram significância estatística quanto a presença de cães em casa e brincar com cães e gatos. Teixeira et al.(2006); Paludo et al. (2007); Roldán et al. (2009); Sharif et al. (2010), analisaram a variável contato com areia na escola ou creche e praças e a consideraram um fator de risco para 
MELLO, I.N.K. e PIETRALONGA, P.A.G. Toxocaríase em crianças e adolescentes: uma revisão sistemática dos estudos epidemiológicos. PUBVET, Londrina, V. 5, N. 31, Ed. 178, Art. 1203, 2011.

soroprevalência positiva, indicando a contaminação frequente de solos e areia por esse agente.

Marmor et al. (1987); Sadjjadi et al. (2000); Roldán et al. (2009) avaliaram geofagia como uma variável de fator de risco para toxocaríase e encontraram respectivamente, o risco relativo (RR) de 3,14 e razão de chances (odds ratio, OR) de 4,54 e 2,6. Nesse sentido outras variáveis foram consideradas para a compreensão dos fatores de risco como Onicofagia (ALDERETE et al., 2003); Presença de quintal de terra (ALDERETE et al. 2003; COELHO et al. 2004) não lavar as mãos antes de comer (SHARIF et al. 2010); Presença de jardins em casa; Brincar no jardim de casa; Brincar em parques públicos (SADJJADI et al. 2000; ROLDÁN et al. 2009) sendo todas significantemente positivas.

Os sintomas como eosinofilia, febre, pneumonia, tosse, problemas gastrointestinais, enxaqueca, dor abdominal, dor nas articulações e erupções cutâneas não puderam ser relacionada com a presença de anticorpos antiToxocara (PALUDO et al. 2007). No entanto Baboolal e Rawlins (2002) associaram sinais clínicos de eczema, crises convulsivas e tosse crônica a sorologia positiva. Ainda nesse sentido, Alderete et al. (2003) e Roldán et al. (2009), identificaram como significativamente positivos episódios anteriores de sibilância, e presença de tosse seca com razao de chance (OR) de 2,49 e 2,79, respectivamente.

Correa e Bismarck (2010) em um estudo de coorte de 72 crianças que apresentaram resultado soronegativo foram acompanhadas por um ano. Um total de cinco crianças infectadas em 801 crianças meses de seguimento, o que significou uma taxa de incidência de 7,63 crianças infectadas por $T$. canis, todas as crianças infectadas eram assintomáticas no final do estudo. E ainda, Lopez et al.(2007) descreveram que $77,8 \%$ dos casos de infecções se apresentavam assintomáticos e além disso constatou casos de larva migrans ocular $(6,7 \%)$ e larva migrans visceral $(15,5 \%)$. 
Tabela 2. Fatores de riscos associados a toxocaríase de 1987 a 2010 .

\begin{tabular}{|c|c|c|c|}
\hline Países & $\mathrm{n}^{\circ}$ de crianças & Idade & Fatores de risco \\
\hline \multicolumn{4}{|l|}{ Brasil } \\
\hline Alderete et al. (2003) & 494 & 7 a 16 anos & $\begin{array}{l}\text { Onicofagia (OR: 2,29); Presença de quintal de terra (OR: } 1,71 \text { ); Renda } \\
\text { familiar menor ou igual a cinco salários mínimos (OR: } 0,85)\end{array}$ \\
\hline Paludo et al. (2007) & 450 & $\begin{array}{l}\text { Sete meses a } 12 \\
\text { anos }\end{array}$ & Gatos em casa (OR: 1.95); Areia na escola (OR: 1.93) \\
\hline Teixeira et al. (2006) & 242 & 1 a 15 anos & $\begin{array}{l}\text { Presença de cães e gatos (OR: } 3,57 \text { ); Contato com areia em escolas (OR: } \\
7,94) \text {. }\end{array}$ \\
\hline \multicolumn{4}{|l|}{ Irã } \\
\hline Sadjjadi et al.(2000) & 519 & 6 a 13 anos & $\begin{array}{l}\text { Local de residência (OR: 0,59); Geofagia (OR: 4,54); grau de escolaridade } \\
\text { dos pais (OR: } 1.07 \text { ) }\end{array}$ \\
\hline Sharif et al.(2010) & 1210 & 7 a 14 anos & $\begin{array}{l}\text { Contato com cães (OR: } 0.36 \text { ); contato com areia (OR: } 0.13 \text { ); não lavar as } \\
\text { mãos antes de comer (OR: } 1.78 \text { ) }\end{array}$ \\
\hline \multicolumn{4}{|l|}{ Peru } \\
\hline Roldán et al.(2009) & 200 & 5 a 12 anos & $\begin{array}{l}\text { Presença de jardins em casa (OR: } 1,82) \text {; Brincar no jardim de casa (OR: } \\
\text { 3,25); Brincar em parques públicos (OR: 1,78); Presença de animais em } \\
\text { áreas públicas (OR: } 2,35 \text { ); História de geofagia (OR:2,67) }\end{array}$ \\
\hline \multicolumn{4}{|l|}{ Estados Unidos } \\
\hline Marmor et al. (1987) & $\begin{array}{l}155 \text { pares caso- } \\
\text { controle }\end{array}$ & 1 a 15 anos & Geofagia $(R R=3,14)$; presença de cachorros em casa $(R R=5,22)$ \\
\hline
\end{tabular}


MELLO, I.N.K. e PIETRALONGA, P.A.G. Toxocaríase em crianças e adolescentes: uma revisão sistemática dos estudos epidemiológicos. PUBVET, Londrina, V. 5, N. 31, Ed. 178, Art. 1203, 2011.

\section{DISCUSSÃO}

Todos os estudos selecionados para a presente revisão (com exceção do trabalho de Correa e Bismarck, 2010 e Marmor et al. 1987) foram realizados utilizando delineamento do tipo transversal, também chamados estudos de prevalência que se caracterizam pela observação direta de determinada quantidade planejada de indivíduos em uma única oportunidade. Em todos os trabalhos analisados, os pesquisadores utilizaram testes sorológicos para determinação da prevalência de anticorpos anti-Toxocara nas populações. A grande utilização deste delineamento em estudos epidemiológicos pode ser justificada pelo baixo custo de sua execução e também por ser o delineamento mais indicado para determinar a presença de antígenos, anticorpos e outros marcadores biológicos no sangue coletado de indivíduos nos chamados estudos soroepidemiológicos.

A diversidade entre as variáveis utilizadas para medir os fatores de riscos e as diferentes faixas etárias empregadas nos estudos analisados foram limitações metodológicas importantes que nos impediram de estabelecer uma real associação entre as soroprevalência de toxocaríase e crianças, assim como limitaram a comparação entre os estudos. Ainda outro fator limitante em nossa revisão sistemática foi à falta de trabalhos em âmbito nacional tanto no Brasil quanto em outros países, uma vez que, os estudos se apresentaram por regiões. Ainda observamos que a maior parte dos estudos apresentava como base populacional escolares e usuários de serviço hospitalar de instituições públicas, excluindo assim a população que não utiliza esses serviços e não caracterizando adequadamente a população.

A maioria dos estudos utilizou questionários como instrumento para medir as variáveis através de dados clínicos e epidemiológicos a fim de avaliar possíveis fatores de risco para toxocaríase. Dados epidemiológicos incluíam: hábitos de onicofagia, geofagia, presença de gatos ou cães em casa; exposição a solos, grama e caixa de areia, local onde a criança geralmente brinca (escola, 
creche e praças publicas), renda familiar, idade, sexo, localização da casa e dados clínicos: bronquite, asma, febre, pneumonia, resfriado, tosse, diarréia e vômitos, cefaléia, prurido generalizado ou eczema, ou dor nos membros inferiores. Os autores relataram que a aplicação dos questionários aos pais e responsáveis foi de forma confidencial e individual, entretanto, não se pode descartar a possibilidade de ter ocorrido vieses de informação.

Em nossa revisão sistemática observamos variação na prevalência de toxocaríase em crianças de 8,7 a $38,8 \%$ nos estudos realizados no Brasil e de 25 a 67\% nos estudos selecionados realizados em outros países (Argentina, Irã, Trinidade, Peru e Croácia). Acreditamos que esta ampla variação seja decorrente da diferença de características das populações amostrais.

Observamos ainda que há falta de padronização na elaboração de estudos epidemiológicos para toxocaríase, que permitam que os dados sejam consistentes para garantir a realização de comparações confiáveis entre os dados.

\section{CONCLUSÕES}

A revisão bibliográfica mostrou que as pesquisas no assunto devem melhorar sua qualidade metodológica, incluindo a padronização de estudos epidemiológicos, a fim de tornar a comparação dos resultados das pesquisas de diferentes regiões confiável e análise para ajuste dos possíveis fatores de confundimento para identificar os fatores de risco.

Os resultados observados nos estudos reafirmam a importância que as autoridades de saúde devem atribuir a essa infecção particularmente nas regiões desfavorecidas socioeconomicamente, uma vez que a toxocaríase não é reconhecida como problema de saúde publica. Por fim, os esforços de promoção de saúde devem ser orientados na conscientização da população sobre os riscos associados com o potencial zoonótico da doença e as formas de minimizá-los. 


\section{REFERÊNCIAS}

ALDERETE, J.M.S.et al. Prevalence of Toxocara Infection in Schoolchildren from the Butantã Region, São Paulo, Brazil. Memórias do Instituto Oswaldo Cruz, Rio de Janeiro, v. 98, n.5, p. 593-597, 2003.

ASHWATH, M. L.; ROBINSON, D. R.; KATNER, H. P. A presumptive case of toxocariasis associated with eosinophilic pleural effusion: case report and literature view. American Journal of Tropical Medicine and Hygiene, Estados Unidos, v. 71, p. 764, 2004.

AZAR, D. M.; MARTIN, F. Pediatric uveitis: a Sydney clinic experience. Clinical Experimental Ophthalmology, Australia, v. 32, p. 468-471, 2004.

BABOOLAL, S.; RAWLINS, S.C. Seroprevalence of toxocariasis in schoolchildren in Trinidad. Transactions of the Royal Society of Tropical Medicine and Hygiene, Londres, v. 96, p.139-143, 2002.

BÄCHLI, H.; MINET, J. C.; GRATZL, O. Cerebral toxocariasis: a possible cause of epileptic seizure in children. Children Nervous System, Estados Unidos, v. 20, p. 468-472, 2004.

CAMPOS JUNIOR, D.; ELEFANT, G. R.; SILVA, E. O. M. Freqüência de soropositividade para antígenos de Toxocara canis em crianças de classes sociais diferentes. Revista do Instituto de Medicina Tropical de São Paulo, São Paulo, v.36, n.4, p. 509-513, 2003.

CHIEFFI, P. P. et al. Visceral larva migrans: a soroepedemiological survey in Five municipalities of São Paulo State, Brazil. Revista do Instituto de Medicina Tropical de São Paulo, São Paulo, v. 32, p. 204-210, 1990.

COELHO, L. M.P.S. et al. Human Toxocariasis: a Seroepidemiological Survey in Schoolchildren of Sorocaba, Brazil. Memórias do Instituto Oswaldo Cruz, Rio de Janeiro, v. 99, n.6, p.533557, 2004.

CORREA, C.R.S.; BISMARCK, C.M. Toxocariasis: Incidence, Prevalence and the Time Serum remains Positive in School Children from Campinas, SP, Brazil. Journal of Tropical Pediatrics, Oxford, v. 56, N. 3, p.215-216, 2010.

DAMIAN, M. M. et al. Freqüência de anticorpo anti-Toxocara canis em comunidade do Rio Uatumã, no Estado do Amazonas. Revista do Instituto de Medicina Tropical de São Paulo, São Paulo, v. 40, p. 661-664, Nov./dez, 2007.

FAN, C. K. et al. Seroepidemiology of Toxocara canis infection among mountain aboriginal schoolchildren living in contamined districts in eastern Taiwan. Tropical Medicine and International Health, Londres, v. 9, p. 1312-1318, 2004.

GAVIGNET, B. et al. Cutaneous manifestation of human toxocariasis. Journal American Academy of Dermatology, Estados Unidos, v. 59, p. 1031-1042, 2008.

KEEGAN, J. D.; HOLLAND, C. V. Contamination of the hair of owned dogs whith the eggs of Toxocara spp. Veterinary Parasitology, Estados Unidos, v.173, p.161-164,2010.

LOPEZ, M.A. et al. Toxocariosis en niños de una region subtropical. Medicina, Buenos Aires, v. 65, p.226-230, 2005.

MARMOR, M. et al. Toxocara canis Infection of Children: Epidemiologic and Neuropsychologic Findings. American Journal of Public Health, Estados Unidos, v. 77, n. 5, 1987.

MOREIRA-SILVA, S. F. et al. Toxocariasis of the Central Nervous System: whith report of two cases. Revista do Instituto de Medicina Tropical de São Paulo, São Paulo, v. 37, p. 169$174,2004$. 
PALUDO, M. L. et al. Frequency of Toxocara infection in children attended by the health public service of maringá, south Brazi. Revista do Instituto de Medicina Tropical de São Paulo, São Paulo, v. 49, n.6, p.343-348, 2007.

PAWLOWSKI, Z. Toxocariasis in humans: clinical expression and treatment dilemma. Journal of Helminthology, Reino Unidos, v. 75, p. 299-305, 2001.

RAYES, A. A. et al. Human toxocariasis and pyogenic liver abscess: A possible association. The American Journal of Gastroenterology, Chicago, v. 96, p. 563-566, 2001.

REY, L. Parasitologia: Parasitos e doenças parasitárias do homem nos trópicos ocidentais. Rio de janeiro: Guanabara Koogan, 2008 p. 562.

ROLDÁN, W. H. et al. Frequency of eosinophilia and risk factors and their association with toxocara infection in schoolchildren during a health survey in the north of Lima, Peru. Revista do Instituto de Medicina Tropical de São Paulo, São Paulo, v. 50, n.5, p.273-278, 2008.

SADJJADI, S.M. et al. Seroprevalence of Toxocara infection in school children in Shiraz, Southern Iran. Journal of Tropical Pediatrics, Oxford, v.46, p.327-330, 2000.

SALEM, G.; SCHANTZ, P. Toxocaral visceral larva migrans after ingestion of raw lamb liver. Clinical Infectious Diseases, Oxford, v. 15, p. 743-744, 1992.

SCHANTZ, P. Toxocara larva migrans now. American Journal Tropical Medicine and Hygiene, Estados Unidos, v. 41, p. 21-34, 1989.

SHARIF, M. et al. Seroprevalence of Toxocariasis in Schoolchildren in Northern Iran. Pakistan Journal of Biological Sciences, Paquistão, v.13, n.4, p.180-184, 2010.

STÜCHLER, D.; WEISS, N.; GASSMAN, M. Transmission of Toxocariasis. Journal of Infectious Diseases, Oxford, v. 162, p. 571, 1990.

SVIBEN, M., et al. Seroprevalence of Toxocara canis infection among asymptomatic children with eosinophilia in Croatia. Journal of Helminthology, Reino Unido, v.83, p.369-371, 2009.

TEIXEIRA, C. R. et al. Frequency and risk factors for toxocariasis in children from a pediatric outpatient center in southeastern Brazil. Revista do Instituto de Medicina Tropical de São Paulo, São Paulo, v. 48, n.5, p.251-255, 2006.

TONELLI, E. Toxocariasis and asthma: a relevant association. Jornal de Pediatria, Rio de Janeiro, v. 81, p. 95-96, 2005.

VIDAL, J. E.; SZTCJNBOK, J.; SEGURO, A. C. Eosinophilic meningoencephalitis due to Toxocara canis: case report and review of the literature. Journal of Tropical Medicine, Estados Unidos, v. 69, p. 341-343, 2003.

WOLFE, A.; WRIGHT, I. P. Parasitic nematode eggs in fur samples from dogs. Veterinary record ,Londres, v. 154, n. 13, p. 408, 2004. 